\title{
Optimal Range and Beamwidth for Radar Tracking of Maneuvering Targets Using Nearly Constant Velocity Filters
}

Per Boström-Rost, Daniel Axehill, William Dale Blair and Gustaf Hendeby

The self-archived postprint version of this journal article is available at Linköping University Institutional Repository (DiVA):

http:/ / urn.kb.se/ resolve?urn=urn:nbn:se:liu:diva- 166532

N.B.: When citing this work, cite the original publication.

Boström-Rost, P., Axehill, D., Blair, W. D., Hendeby, G., (2020), Optimal Range and Beamwidth for Radar Tracking of Maneuvering Targets Using Nearly Constant Velocity Filters, Proceedings of the 2020 IEEE Aerospace Conference. https:// doi.org/ 10.1109/AERO47225.2020.9172558

Original publication available at:

https:// doi.org/ 10.1109/AER047225.2020.9172558

Copyright:

Institute of Electrical and Electronics Engineers (IEEE)

http:/ / www.ieee.org/ index.html

(C) 2018 IEEE

Personal use of this material is permitted. However, permission to reprint/ republish this material for advertising or promotional purposes or for creating new collective works for resale or redistribution to servers or lists, or to reuse any copyrighted component of this work in other works must be obtained from the IEEE 


\section{Optimal Range and Beamwidth for Radar Tracking of Maneuvering Targets Using Nearly Constant Velocity Filters}

\author{
Per Boström-Rost \\ Linköping University \\ Linköping, Sweden \\ per.bostrom-rost@liu.se
}

\author{
Daniel Axehill \\ Linköping University \\ Linköping, Sweden \\ daniel.axehill@liu.se \\ Gustaf Hendeby \\ Linköping University \\ Linköping, Sweden \\ gustaf.hendeby@liu.se
}

\author{
William Dale Blair \\ Georgia Tech Research Institute \\ Georgia Institute of Technology \\ Atlanta, Georgia 30332 \\ dale.blair@gtri.gatech.edu
}

\begin{abstract}
For a given radar system on an unmanned air vehicle, this work proposes a method to find the optimal tracking range and the optimal beamwidth for tracking a maneuvering target. An inappropriate optimal range or beamwidth is indicative of the need for a redesign of the radar system. An extended Kalman filter (EKF) is employed to estimate the state of the target using measurements of the range and bearing from the sensor to the target. The proposed method makes use of an alpha-beta filter to predict the expected tracking performance of the EKF. Using an assumption of the maximum acceleration of the target, the optimal tracking range (or beamwidth) is determined as the one that minimizes the maximum mean squared error (MMSE) of the position estimates while satisfying a userdefined constraint on the probability of losing track of the target. The applicability of the design method is verified using Monte Carlo simulations.
\end{abstract}

\section{TABLE OF CONTENTS}

1. INTRODUCTION..............................

2. Tracking With Range-Bearing SENSORS ......2

3. DESIGN OF NCV Kalman Filters fOR TraCKing Maneuvering Targets $\ldots \ldots \ldots \ldots \ldots \ldots \ldots .2$

4. Design of Tracking Filters for RangeBEARING SENSORS ............................ 3

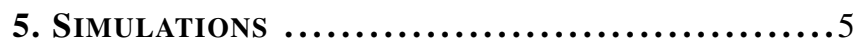

6. Conclusions ................................ 7

ACKNOWLEDGMENTS ............................7

REFERENCES .................................... 7

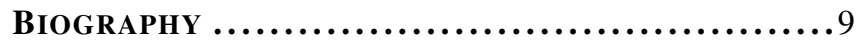

\section{INTRODUCTION}

When tracking a maneuvering target using a mobile rangebearing sensor with a limited field of $\mathrm{v}$ iew, $\mathrm{t}$ he following question arises: should the tracking range be small to avoid large estimation errors in the cross-range direction or should the tracking range be large to reduce the risk of losing track of the target as it maneuvers? A related issue arises in the case of a stationary radar with variable beamwidth: increasing the beamwidth reduces the risk of losing track of the target, but it also decreases the signal-to-noise ratio.

A standard approach in target tracking is to employ a Kalman filter variant to estimate the state of the target. In active target tracking or sensor management, the available sensors are actively controlled in order to improve the estimation performance [1], [2]. A measure of the performance is thus needed, and a common choice is the covariance matrix of the state estimates of the Kalman filter, used e.g. in [3]. A nearly constant velocity model (NCV) is often used to describe the motion of the target, and the acceleration is modeled as additive white Gaussian process noise. However, when the target is maneuvering, the actual acceleration is deterministic rather than random. This means that the covariance of the state estimate is not always suitable as a measure of the tracking performance. An alternative measure of the tracking performance is the maximum mean squared error (MMSE) of the state estimates, which also includes a bias term.

A method to select the process noise standard deviation in an NCV Kalman filter to minimize the MMSE of the filtered estimates was proposed in [4]. In [5], the method was extended to radar tracking filters, where the measurements are nonlinear functions of the target state. The method was further extended to allow for minimization of the MMSE of the predicted estimates in [6].

Methods for active target tracking based on graph search algorithms, e.g., [7], [8], allow for efficient planning in multitarget scenarios, but those methods have been shown to lead to greedy behavior and lost tracks in the case of single-target tracking [9]. This paper focuses on the problem of active tracking of a single target using a range-bearing sensor with limited field of view and utilizes the design strategies of [4], [5], [6] to find the optimal range and optimal beamwidth for tracking given an upper bound on the acceptable risk of losing track of the target.

This paper is organized as follows. The problem of tracking maneuvering targets using a range-bearing sensor is outlined in Section 2. Section 3 summarizes the design methods developed in [4], [6]. Section 4 proposes methods to find optimal tracking parameters for range-bearing tracking, and those methods are evaluated using Monte Carlo simulations in Section 5. Finally, the concluding remarks are given in Section 6. 


\section{Tracking With RANGe-BEARING SENSORS}

The problem considered in this paper is tracking of a maneuvering target using a range-bearing sensor with limited field of view, e.g., a radar. Many radar systems make use of Kalman filter variants to estimate the state of the target. Often, a nearly constant velocity (NCV) model is used to predict the motion of the target. In the two-dimensional case, the corresponding state vector is given by

$$
x=\left[\begin{array}{llll}
\mathrm{x}_{1} & \mathrm{v}_{1} & \mathrm{x}_{2} & \mathrm{v}_{2}
\end{array}\right]^{\top},
$$

where $\left(\mathrm{x}_{1}, \mathrm{x}_{2}\right)$ represents the position of the target in Cartesian coordinates and $\left(v_{1}, v_{2}\right)$ represents the velocity of the target.

The measurement model for sensors that measure range and bearing is a nonlinear function of the target state and sensor state and given by

$$
\begin{aligned}
y_{k} & =h\left(x_{k}, s_{k}\right)+e_{k}, \quad e_{k} \sim \mathcal{N}\left(0, R_{k}\right), \\
h(x, s) & =\left[\begin{array}{c}
r(x, s) \\
\theta(x, s)
\end{array}\right]=\left[\begin{array}{c}
\sqrt{\left(\mathrm{x}_{1}-\mathrm{s}_{1}\right)^{2}+\left(\mathrm{x}_{2}-\mathrm{s}_{2}\right)^{2}} \\
\arctan \left(\frac{\mathrm{x}_{2}-\mathrm{s}_{2}}{\mathrm{x}_{1}-\mathrm{s}_{1}}\right)
\end{array}\right],
\end{aligned}
$$

where $\left(s_{1}, s_{2}\right)$ is the position of the sensor, $r$ is the range, and $\theta$ is the bearing. The measurement noise $e_{k}$ is assumed to be Gaussian distributed with zero mean and covariance matrix $R_{k}$, which is assumed to be diagonal (i.e., the noise in the range measurement is independent from the noise in the bearing measurement):

$$
R_{k}=\left[\begin{array}{cc}
\sigma_{r}^{2} & 0 \\
0 & \sigma_{\theta}^{2}
\end{array}\right]
$$

A monopulse radar will be used as an example of a rangebearing sensor in this paper. The standard deviation of the bearing measurement noise $\sigma_{\theta}$ is then proportional to the beamwidth $\theta_{\mathrm{BW}}$. Assuming that measurements are on boresight, the variance of the bearing measurement noise can according to [10] be approximated by

$$
\sigma_{\theta}^{2}=\frac{\theta_{\mathrm{BW}}^{2}}{2 k_{m}^{2} \mathrm{SNR}},
$$

where $k_{m}=1.6$ and SNR is the signal-to-noise ratio, for which $17 \mathrm{~dB}$ is a reasonable value [11].

As the measurements are nonlinear functions of the target state, the extended Kalman filter (EKF) [12] is employed to estimate the state.

\section{DESIGN OF NCV KALMAN FILTERS FOR TraCKING MANEUVERING TARgETS}

The Kalman filter [13] estimates the states of a dynamical system based on a linear Gaussian state-space model with additive noise:

$$
\begin{aligned}
x_{k+1} & =F_{k} x_{k}+G_{k} w_{k}, \\
y_{k} & =H_{k} x_{k}+e_{k},
\end{aligned}
$$

where $x_{k}$ is the state vector and $y_{k}$ is the obtained measurement, both at time $k$. The process noise $w_{k}$ and the measurement noise $e_{k}$ are assumed to be mutually independent and Gaussian distributed according to $w_{k} \sim \mathcal{N}\left(0, Q_{k}\right)$ and $e_{k} \sim \mathcal{N}\left(0, R_{k}\right)$, respectively.

The NCV motion model is based on the assumption that the target travels with constant velocity and treats acceleration as process noise. Assuming a discrete white noise acceleration (DWNA) process noise, the elements of (5) for the NCV model defined in a single coordinate are given by

$$
\begin{aligned}
x_{k} & =\left[\begin{array}{ll}
\mathrm{x}_{k} & \mathrm{v}_{k}
\end{array}\right]^{\top}, \\
F_{k} & =\left[\begin{array}{ll}
1 & T \\
0 & 1
\end{array}\right], \\
G_{k} & =\left[\begin{array}{ll}
\frac{1}{2} T^{2} & T
\end{array}\right]^{\top}, \\
Q_{k} & =\sigma_{w}^{2},
\end{aligned}
$$

where $T$ is the time between two consecutive measurements, and $\sigma_{w}^{2}$ is the variance of the acceleration process noise in $\mathrm{m}^{2} / \mathrm{s}^{4}$. The elements of (5) that correspond to measurements of the target's position are defined as

$$
\begin{aligned}
H_{k} & =\left[\begin{array}{ll}
1 & 0
\end{array}\right], \\
R_{k} & =\sigma_{e}^{2},
\end{aligned}
$$

where $\sigma_{e}^{2}$ is the variance of the measurement noise in $\mathrm{m}^{2}$.

The steady-state form of the NCV Kalman filter with measurements of the target's position is the alpha-beta filter. For a filter to achieve steady-state conditions, the noise processes must be stationary and the time between consecutive measurements must be constant. While these conditions are seldom satisfied in practice, the alpha-beta filter can be used to predict the average or expected tracking performance of the NCV Kalman filter. In [4], [6], it was used to develop design methods for the NCV Kalman filter. In particular, a method to find the process noise standard deviation that minimizes the MMSE in the position estimates of a NCV Kalman filter was proposed. As the method will be used also in this paper, it is summarized in this section.

The steady-state gains of the alpha-beta filter are given by

$$
K_{k}=\left[\begin{array}{ll}
\alpha & \frac{\beta}{T}
\end{array}\right]^{\top},
$$

and the filtering equations for the alpha-beta filter are given by

$$
\begin{aligned}
\hat{\mathrm{x}}_{k \mid k-1} & =\hat{\mathrm{x}}_{k-1 \mid k-1}+T \hat{\mathrm{v}}_{k-1 \mid k-1}, \\
\hat{\mathrm{v}}_{k \mid k-1} & =\hat{\mathrm{v}}_{k-1 \mid k-1}, \\
\hat{\mathrm{x}}_{k \mid k} & =\hat{\mathrm{x}}_{k \mid k-1}+\alpha\left(y_{k}-\hat{\mathrm{x}}_{k \mid k-1}\right), \\
\hat{\mathrm{v}}_{k \mid k} & =\hat{\mathrm{v}}_{k \mid k-1}+\frac{\beta}{T}\left(y_{k}-\hat{\mathrm{x}}_{k \mid k-1}\right) .
\end{aligned}
$$

As given in [14], the optimal gains for the DWNA model are computed as

$$
\begin{aligned}
\Gamma_{\text {DWNA }} & =\frac{\sigma_{w} T^{2}}{\sigma_{e}}=\frac{\beta}{\sqrt{1-\alpha}}, \\
\beta & =2(2-\alpha)-4 \sqrt{1-\alpha},
\end{aligned}
$$

where $\Gamma_{\text {DWNA }}$ is the random tracking index, a dimensionless parameter that is proportional to the ratio of the uncertainty due to the target maneuverability and the sensor measurements errors [14]. 
The process noise standard deviation for the NCV Kalman filter that corresponds to the optimal gains $\alpha$ and $\beta$ is given by

$$
\sigma_{w}=\kappa A_{\max }
$$

where $\kappa$ is defined as the ratio between the random and the deterministic tracking index $\Gamma_{\mathrm{D}}$ :

$$
\kappa=\frac{\Gamma_{\text {DWNA }}}{\Gamma_{\mathrm{D}}}=\frac{\sigma_{w}}{A_{\max }} .
$$

The deterministic tracking index [4] is defined as

$$
\Gamma_{\mathrm{D}}=\frac{A_{\mathrm{max}} T^{2}}{\sigma_{e}}
$$

where $A_{\max }$ is the maximum acceleration of the target. The fact that it depends on the maximum acceleration of the target makes the deterministic tracking index more suitable than the random tracking index for characterizing deterministic-type maneuvers [4].

According to [6], the MMSE in the one-step predicted position estimates of the alpha-beta filter is given by

$$
\operatorname{MMSE}_{p+}\left(\alpha, \beta, \Gamma_{\mathrm{D}}, \sigma_{e}\right)=\sigma_{e}^{2}\left[\frac{2 \alpha^{2}+\beta(2+\alpha)}{\alpha(4-2 \alpha-\beta)}+\frac{1}{\beta^{2}} \Gamma_{\mathrm{D}}^{2}\right] .
$$

For a given value of the deterministic tracking index $\Gamma_{D}$, the process noise standard deviation $\sigma_{w}^{\max }$ that minimizes the MMSE in the one-step predicted position estimates can be computed by first solving

$$
\begin{aligned}
\kappa^{\max }\left(\Gamma_{\mathrm{D}}\right)=\underset{\kappa}{\arg \min } & \operatorname{MMSE}_{p+}\left(\alpha, \beta, \Gamma_{\mathrm{D}}, \sigma_{e}\right) \\
\text { subject to } & \beta=2(2-\alpha)-4 \sqrt{1-\alpha} \\
& \Gamma_{\text {DWNA }}=\beta / \sqrt{1-\alpha} \\
& \kappa=\Gamma_{\text {DwNA }} / \Gamma_{\mathrm{D}},
\end{aligned}
$$

and then computing

$$
\sigma_{w}^{\max }=\kappa^{\max } A_{\max } .
$$

Note that $\sigma_{e}$ is a scaling factor in (14) and that $\kappa^{\max }$ thus is a function of the deterministic tracking index $\Gamma_{\mathrm{D}}$, as illustrated in Figure 1.

Using the constraint that the MMSE in the one-step predicted position estimates should not exceed the measurement noise variance gives that $\mathrm{MMSE}_{p+} \leq \sigma_{e}^{2}$, which, using (14), implies that

$$
\frac{2 \alpha^{2}+\beta(2+\alpha)}{\alpha(4-2 \alpha-\beta)}+\frac{1}{\beta^{2}} \Gamma_{\mathrm{D}}^{2} \leq 1 .
$$

For a given value of $\Gamma_{\mathrm{D}}$, let $\alpha^{\text {min }}$ denote the smallest $\alpha$ that defines $\beta^{\text {min }}$ through (10b) and satisfies (17) with equality. These gains define a random tracking index $\Gamma_{\mathrm{DWNA}}^{\mathrm{min}}$ through (10a), which using (11) gives the corresponding process noise standard deviation as

$$
\sigma_{w}^{\min }=\frac{\Gamma_{\mathrm{DWNA}}^{\min }}{\Gamma_{\mathrm{D}}} A_{\max }=\kappa^{\min } A_{\max }
$$

where $\kappa^{\text {min }}$ is a function of the deterministic tracking index $\Gamma_{\mathrm{D}}$, as illustrated in Figure 1. The value $\sigma_{w}^{\min }$ is the smallest

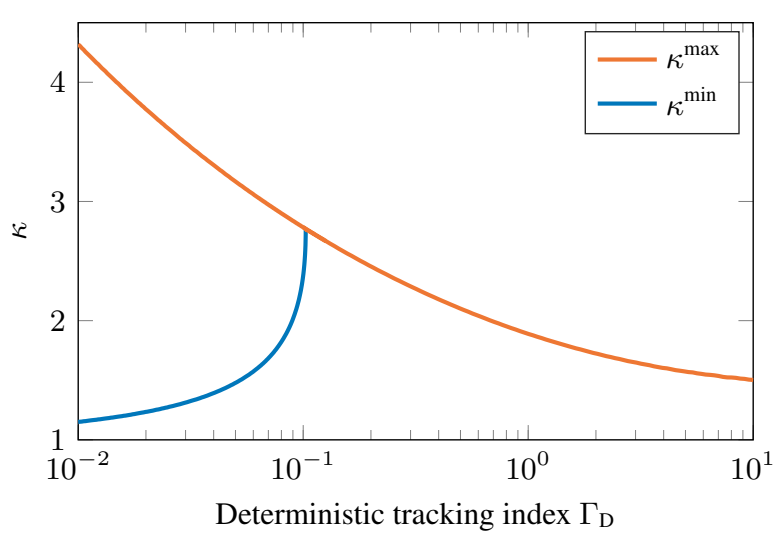

Figure 1: Minimum and maximum values of $\kappa$ for selection of process noise variance.

process noise standard deviation that can be used in order to satisfy the constraint that the MMSE in the one-step predicted position estimates should not exceed the measurement noise variance. Note that for $\Gamma_{\mathrm{D}}>0.1$, there are no designs for the NCV Kalman filter that satisfy this constraint.

With these design methods, the process noise standard deviation used in an NCV Kalman filter can be selected such that the root mean squared error (RMSE) in the one-step predicted position estimates is approximately equal to the standard deviation of the measurement noise (using $\sigma_{w}^{\min }$ ), or such that the MMSE is minimized during maneuvers (using $\sigma_{w}^{\max }$ ).

\section{Design of Tracking Filters For RANGE-BEARING SENSORS}

The problem of selecting tracking parameters for a rangebearing sensor that is tracking a maneuvering target is considered in this section. We seek to select the parameters such that a performance measure is optimized under the constraint that the probability of losing track of the target is less than a user-defined value, $\gamma$. This can be formulated as the following optimization problem:

$$
\begin{array}{ll}
\text { minimize } & \mathrm{MMSE}_{p+} \\
\text { subject to } & p(\text { lost track }) \leq \gamma
\end{array}
$$

where the objective function $\mathrm{MMSE}_{p+}$ is the MMSE in the one-step predicted position estimates. The optimization variables that are considered are the tracking range, the beamwidth, and the process noise standard deviation used in the EKF.

Motivated by the fact that the measurement noise is independent between the different coordinates of the sensor frame, and that the measurement noise variance in each coordinate tends to be constant or vary slowly, the expected tracking performance in each sensor coordinate can be estimated using an alpha-beta filter [15], [5]. This means that the design methods from [4], [6], that are summarized in Section 3, can be applied also in this case, and the performance of EKFs for range-bearing sensors can be analyzed in the range and crossrange directions separately. In the following, it is assumed that the measurement error (in meters) is greater in the crossrange direction than in the range direction. 


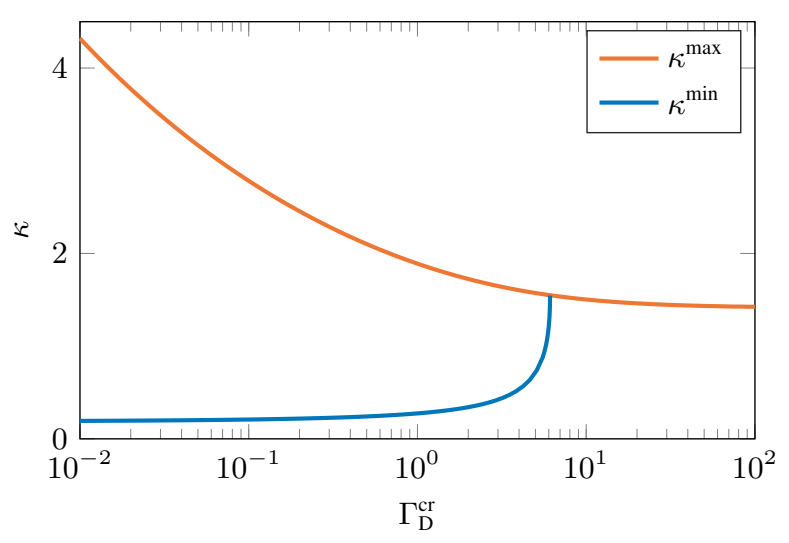

Figure 2: Minimum and maximum values of $\kappa$ for selection of process noise variance.

\section{Filter tuning}

In the cross-range direction, the measurement error in meters for a sensor that measures range and bearing to the target as in (2) is a function of the range. Using the small angle approximation, the standard deviation of the measurement noise in meters is given by

$$
\sigma_{e}^{\mathrm{cr}}=r \sigma_{\theta}
$$

This implies that the corresponding deterministic tracking index in (13) now depends on the range as

$$
\Gamma_{\mathrm{D}}^{\mathrm{cr}}=\frac{A_{\mathrm{max}} T^{2}}{r \sigma_{\theta}} .
$$

Thus, the process noise standard deviation that minimizes the MMSE in the cross-range direction of the one-step predicted position estimates is given by

$$
\sigma_{w}^{\max }=\kappa^{\max }\left(\Gamma_{\mathrm{D}}^{\mathrm{cr}}\right) A_{\max }
$$

where $\kappa^{\max }\left(\Gamma_{D}^{\mathrm{cr}}\right)$ is obtained by solving the parametric program (15). Figure 2 shows $\kappa^{\max }$ as a function of the deterministic tracking index $\Gamma_{\mathrm{D}}^{\mathrm{cr}}$.

As the considered range-bearing sensor has a limited field of view or beamwidth, it needs to be pointed in the right direction in order not to lose track of the target. Assuming that the pointing direction is determined based on the onestep predicted bearing estimates, this means that we need to ensure that the error in the bearing estimates is sufficiently small. Using the small-angle approximation, the MMSE of the bearing estimates is related to the MMSE of the position estimates in the cross-range direction as

$$
\begin{aligned}
\operatorname{MMSE}_{\theta+} & =\frac{1}{r^{2}} \operatorname{MMSE}_{p+}^{\mathrm{cr}} \\
& =\sigma_{\theta}^{2}\left[\frac{2 \alpha^{2}+\beta(2+\alpha)}{\alpha(4-2 \alpha-\beta)}+\frac{1}{\beta^{2}}\left(\Gamma_{\mathrm{D}}^{\mathrm{cr}}\right)^{2}\right] .
\end{aligned}
$$

Assuming Gaussian distributed errors and unbiased estimates, the probability of an error being greater than a given multiple of the standard deviation can be computed. Here, we will use the fact that $99.7 \%$ of the one-step predicted bearing estimates are within three standard deviations of the true value. Hence, defining the largest acceptable RMSE in the one-step predicted bearing estimates, denoted $\tilde{\theta}_{\max }$, as one third of the sensor's beamwidth corresponds to $\gamma=0.003$ in (19). The constraint that limits the probability of losing track of the target is thus captured by

$$
\operatorname{MMSE}_{\theta+} \leq \tilde{\theta}_{\max }^{2}
$$

where $\tilde{\theta}_{\max }=\theta_{\mathrm{BW}} / 3$.

By using (10b) in (23), the $\mathrm{MMSE}_{\theta+}$ is a function of $\alpha$ and $\Gamma_{\mathrm{D}}^{\mathrm{cr}}$. For a given value of $\Gamma_{\mathrm{D}}^{\mathrm{cr}}$, let $\alpha^{\mathrm{min}}$ denote the smallest $\alpha$ for which (24) is satisfied with equality. The corresponding $\beta^{\text {min }}$ is obtained through (10b). Then, the smallest acceptable process noise standard deviation is determined as:

$$
\begin{aligned}
\Gamma_{\mathrm{DWNA}}^{\min } & =\frac{\beta^{\min }}{\sqrt{1-\alpha^{\mathrm{min}}}}, \\
\kappa^{\min } & =\frac{\Gamma_{\mathrm{DWNA}}^{\min }}{\Gamma_{\mathrm{D}}^{\mathrm{cr}}}, \\
\sigma_{w}^{\min } & =\kappa^{\min } A_{\max } .
\end{aligned}
$$

The $\kappa^{\text {min }}$ corresponding to $\tilde{\theta}_{\max }=\theta_{\mathrm{BW}} / 3$ is plotted as a function of $\Gamma_{\mathrm{D}}^{\mathrm{cr}}$ in Figure 2. Note that for values of the deterministic tracking index $\Gamma_{\mathrm{D}}^{\mathrm{cr}}$ greater than six, there is no value of the process noise standard deviation that satisfies the accuracy requirement on one-step predicted bearing estimates.

\section{Optimal tracking range}

Consider the case when the measurement rate $T$, the maximum acceleration of the target $A_{\mathrm{max}}$, the beamwidth $\theta_{\mathrm{BW}}$, and the bearing measurement noise standard deviation $\sigma_{\theta}$ are known. The deterministic tracking index is then a function of the tracking range, which implies that for each tracking range, there is a process noise standard deviation that minimizes the MMSE of the one-step predicted position estimates in the cross-range direction, and a smallest acceptable process noise standard deviation:

$$
\begin{aligned}
\sigma_{w}^{\max }(r) & =\kappa^{\max }(r) A_{\max }, \\
\sigma_{w}^{\min }(r) & =\kappa^{\min }(r) A_{\max } .
\end{aligned}
$$

It is then possible to find an optimal tracking range with a corresponding process noise standard deviation, such that $\mathrm{MMSE}_{p+}^{\mathrm{cr}}$ is minimized and the constraint on the bearing accuracy (24) is satisfied, by solving

$$
\begin{array}{cl}
\underset{r, \sigma_{w}}{\operatorname{arg~min}} & \operatorname{MMSE}_{p+}\left(\alpha, \beta, \Gamma_{\mathrm{D}}^{\mathrm{cr}}, r \sigma_{\theta}\right) \\
\text { subject to } & \mathrm{MMSE}_{\theta+}\left(\alpha, \beta, \Gamma_{\mathrm{D}}^{\mathrm{cr}}, r \sigma_{\theta}\right) \leq \tilde{\theta}_{\text {max }}^{2} \\
& \beta=2(2-\alpha)-4 \sqrt{1-\alpha} \\
& \Gamma_{\mathrm{DWNA}}=\beta / \sqrt{1-\alpha} \\
& \kappa=\Gamma_{\mathrm{DWNA}} / \Gamma_{\mathrm{D}}^{\mathrm{cr}} \\
& \Gamma_{\mathrm{D}}^{\mathrm{cr}}=A_{\text {max }} T^{2} /\left(r \sigma_{\theta}\right) \\
& \sigma_{w}=\kappa A_{\max } .
\end{array}
$$

Due to the relation between the MMSE in the cross-range direction and bearing (23), the same value of $\kappa$ minimizes both $\mathrm{MMSE}_{p+}^{\mathrm{cr}}$ and $\mathrm{MMSE}_{\theta+}$. Thus, the optimal process noise standard deviation is defined by $\sigma_{w}^{\max }=\sigma_{w}^{\min }$ at the 
optimal tracking range $r^{\star}$, which is the smallest range for which (24) can be satisfied. This means that at the optimal tracking range, the process noise standard deviation that minimizes the MMSE in the cross-range direction of the one-step predicted position estimates also yields one-step predicted bearing estimates with RMSE equal to the largest acceptable error $\tilde{\theta}_{\max }$.

\section{Optimal beamwidth}

When the measurement rate $T$, the maximum acceleration of the target $A_{\max }$, and the tracking range $r$ are known, and the bearing measurement noise standard deviation $\sigma_{\theta}$ is related to the beamwidth $\theta_{\mathrm{BW}}$ according to (4), the deterministic tracking index is a function of the beamwidth. Thus, for each beamwidth, there is a process noise standard deviation that minimizes the MMSE of the one-step predicted position estimates in the cross-range direction, and a smallest acceptable process noise standard deviation:

$$
\begin{aligned}
\sigma_{w}^{\max }\left(\theta_{\mathrm{BW}}\right) & =\kappa^{\max }\left(\theta_{\mathrm{BW}}\right) A_{\max }, \\
\sigma_{w}^{\min }\left(\theta_{\mathrm{BW}}\right) & =\kappa^{\min }\left(\theta_{\mathrm{BW}}\right) A_{\max } .
\end{aligned}
$$

This also implies that it is possible to find an optimal beamwidth, such that the MMSE ${ }_{p+}^{\mathrm{cr}}$ is minimized and the $\mathrm{MMSE}_{\theta+}$ is sufficiently small, i.e., satisfies (24), by solving

$$
\begin{array}{cl}
\underset{\theta_{\mathrm{BW}}, \sigma_{w}}{\arg \min } & \operatorname{MMSE}_{p+}\left(\alpha, \beta, \Gamma_{\mathrm{D}}^{\mathrm{cr}}, r \sigma_{\theta}\left(\theta_{\mathrm{BW}}\right)\right) \\
\text { subject to } & \operatorname{MMSE}_{\theta+}\left(\alpha, \beta, \Gamma_{\mathrm{D}}^{\mathrm{cr}}, r \sigma_{\theta}\left(\theta_{\mathrm{BW}}\right)\right) \leq \tilde{\theta}_{\max }^{2}\left(\theta_{\mathrm{BW}}\right) \\
& \beta=2(2-\alpha)-4 \sqrt{1-\alpha} \\
& \Gamma_{\mathrm{DWNA}}=\beta / \sqrt{1-\alpha} \\
& \kappa=\Gamma_{\mathrm{DWNA}} / \Gamma_{\mathrm{D}}^{\mathrm{cr}} \\
& \Gamma_{\mathrm{D}}^{\mathrm{cr}}=A_{\max } T^{2} /\left(r \sigma_{\theta}\left(\theta_{\mathrm{BW}}\right)\right) \\
& \sigma_{w}=\kappa A_{\max } .
\end{array}
$$

Similarly as for the optimal tracking range, the minimum and maximum process noise standard deviations coincide at the optimal beamwidth $\theta_{\mathrm{BW}}^{\star}$, i.e.,

$$
\sigma_{w}^{\max }\left(\theta_{\mathrm{BW}}^{\star}\right)=\sigma_{w}^{\min }\left(\theta_{\mathrm{BW}}^{\star}\right) .
$$

\section{Simulations}

In this section, the methods for selecting the tracking range and beamwidth are evaluated on a number of radar tracking scenarios. The considered target travels at $300 \mathrm{~m} / \mathrm{s}$ and alternates between flying straight and making $135^{\circ}$ turns with $6 \mathrm{~g}$ of acceleration. The turns are made approximately every $120 \mathrm{~s}$ and last for approximately $12 \mathrm{~s}$ each. The maximum acceleration of the target is $A_{\max }=60 \mathrm{~m} / \mathrm{s}^{2}$. The sensor obtains range and bearing measurements when the target is within field of view, and the state of the target is estimated using an EKF.

\section{Tracking at optimal range}

This set of experiments investigates how the range between sensor and target affects the tracking performance. Consider a mobile radar that is tracking the target with trajectory according to Figure 3 . The radar has a constant beamwidth of $\theta_{\mathrm{BW}}=$ $5^{\circ}$ and measures range with standard deviation of $10 \mathrm{~m}$ and bearing with standard deviation of $\sigma_{\theta}=5.5 \mathrm{mrad}$ every two seconds, i.e., $T=2 \mathrm{~s}$. The bearing measurement accuracy

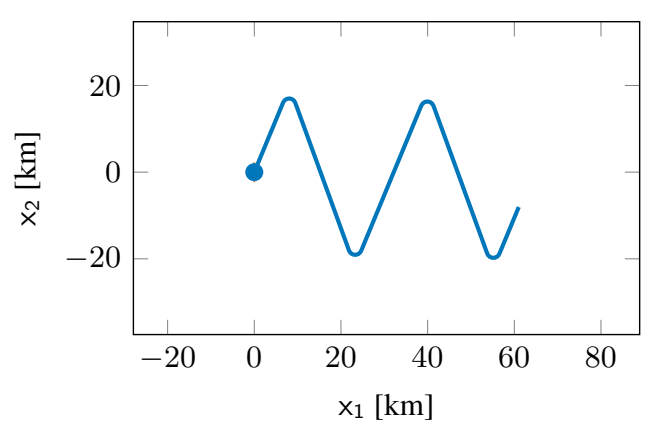

Figure 3: Trajectory of a 6g-maneuvering target that travels at $300 \mathrm{~m} / \mathrm{s}$.

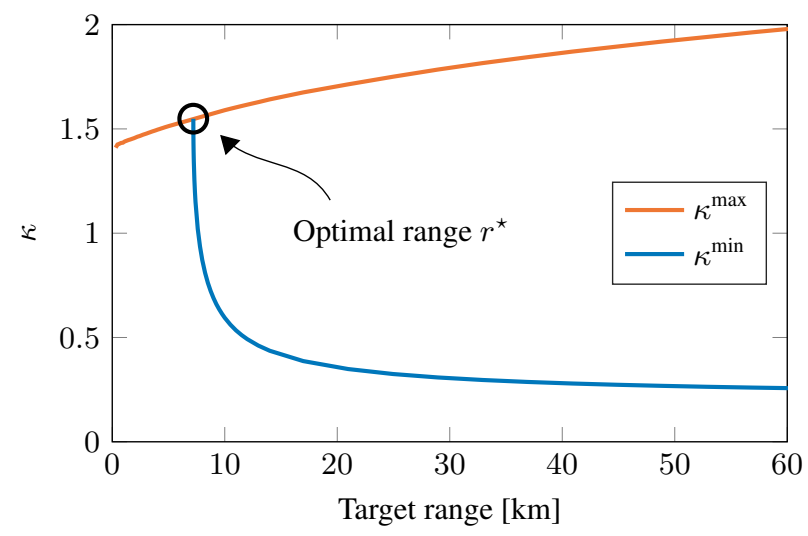

Figure 4: Minimum and maximum values of $\kappa$ for defining the process noise standard deviation in terms the maximum target acceleration.

corresponds to a SNR level of $17 \mathrm{~dB}$ in (4). The accuracy requirement on one-step predicted bearing estimates is set to a third of the radar's beamwidth, i.e., $\tilde{\theta}_{\max }=5 / 3$ degrees.

The deterministic tracking index in the cross-range direction is in this case a function of the variable target range. Figure 4 gives $\kappa^{\max }$ and $\kappa^{\min }$ as functions of target range for the considered scenario, and thus also the process noise standard deviations that minimize $\mathrm{MMSE}_{p+}^{\mathrm{cr}}$ and satisfy the bearing accuracy requirement as functions of the range through (26).

The optimal tracking range, computed by solving (27) numerically, is in this case approximately $7.2 \mathrm{~km}$. At smaller ranges, there is no choice of process noise standard deviation that results in the required accuracy in bearing predictions. At larger ranges, the MMSE of the one-step predicted position estimates is increased.

To evaluate how the choice of tracking range affects the performance, 10000 Monte Carlo simulations were run for three different tracking ranges: a range that is shorter than the optimal one (Case 1), the range that is optimal according to the proposed design method with the corresponding optimal process noise standard deviation (Case 2), and one greater tracking range with both $\sigma_{w}^{\max }$ (Case 3$)$ and $\sigma_{w}^{\min }$ (Case 4$)$. The tracking ranges and process noise standard deviations used in the different cases are summarized in Table 1.

Figure 5 shows the results of the Monte Carlo simulations. For each case, the mobile radar's trajectory is pre-computed 


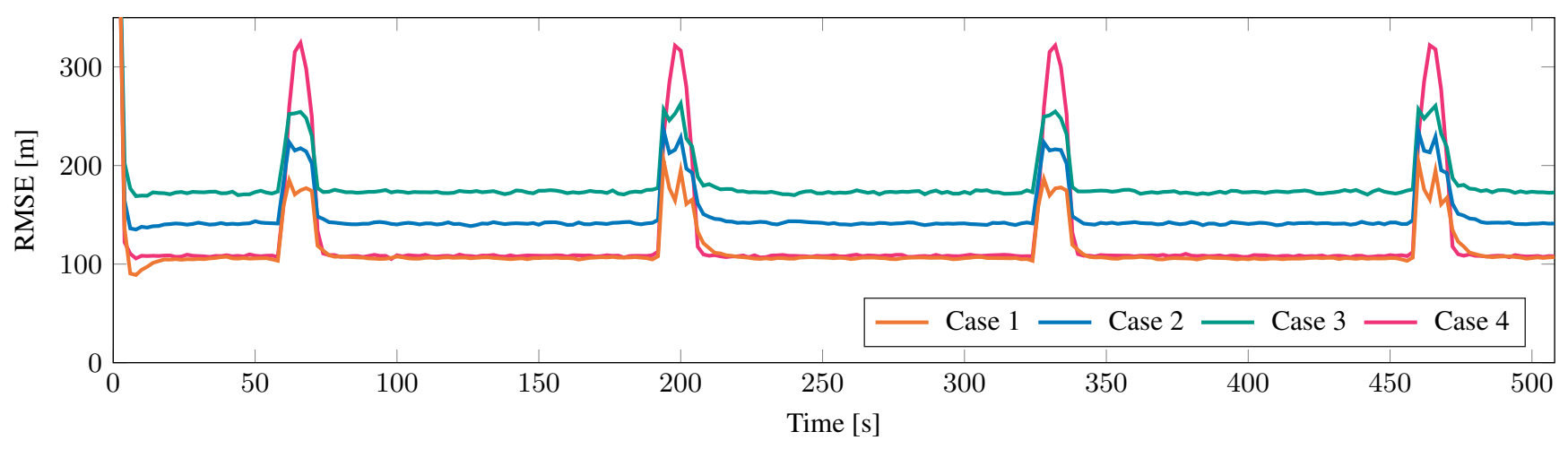

(a) RMSE of the one-step predicted position estimates.

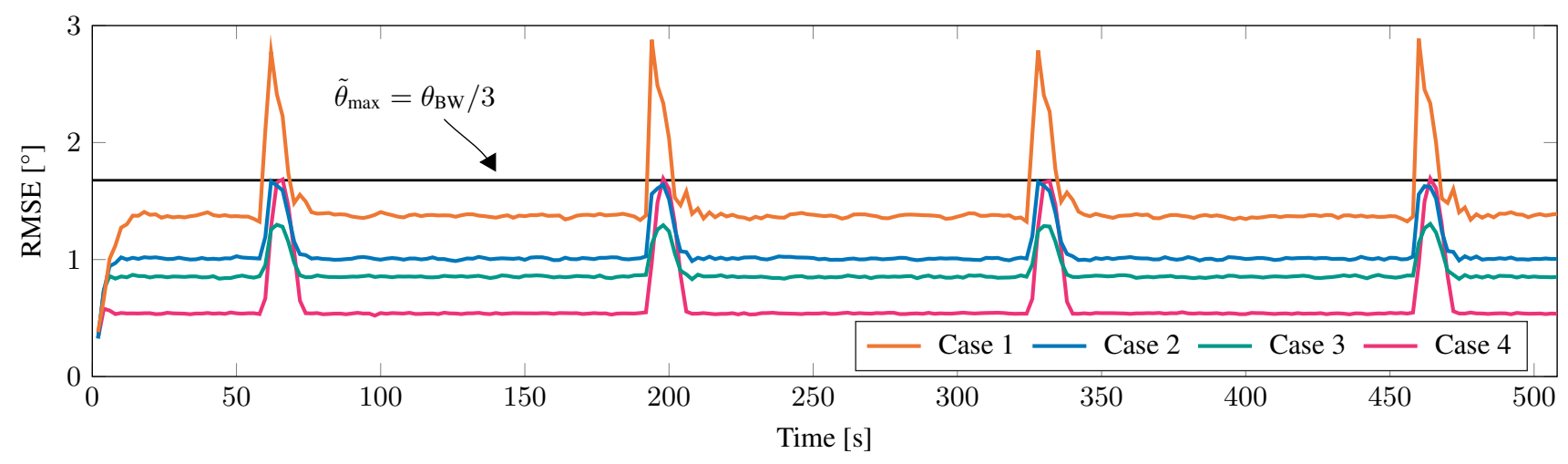

(b) RMSE of the one-step predicted bearing estimates.

Figure 5: Results from 10000 Monte Carlo simulations of the scenario in Figure 3, where the target is tracked by a mobile radar with constant beamwidth of five degrees.

Table 1: Parameters for the mobile radar scenario.

\begin{tabular}{lcc}
\hline Case & Tracking range & Process noise std. dev \\
\hline$\# 1$ & $\frac{1}{2} r^{\star}=3.6 \mathrm{~km}$ & $\sigma_{w}^{\max }\left(\frac{1}{2} r^{\star}\right)$ \\
$\# 2$ & $r^{\star}=7.2 \mathrm{~km}$ & $\sigma_{w}^{\max }\left(r^{\star}\right)=\sigma_{w}^{\min }\left(r^{\star}\right)$ \\
$\# 3$ & $\frac{3}{2} r^{\star}=10.8 \mathrm{~km}$ & $\sigma_{w}^{\max }\left(\frac{3}{2} r^{\star}\right)$ \\
$\# 4$ & $\frac{3}{2} r^{\star}=10.8 \mathrm{~km}$ & $\sigma_{w}^{\min }\left(\frac{3}{2} r^{\star}\right)$ \\
\hline
\end{tabular}

to keep a constant distance to the target, and the beam is pointed toward the estimated target position online. The peaks in the RMSE occur as the target turns with maximum acceleration. The track is considered to be lost if four consecutive measurements are missed. Note that data from simulations where the track was lost were discarded when generating Figure 5.

Figure 6a shows the errors in the one-step predicted position estimates. As expected for this type of sensor, the RMSE increases with the tracking range. Note that $\sigma_{w}^{\max }$ gives lower peak RMSE than $\sigma_{w}^{\min }$ during maneuvers at the cost of higher RMSE when the target follows a straight path.

Figure $6 \mathrm{~b}$ shows the errors in the one-step predicted bearing estimates. When the tracking range is large enough, the use of the minimum acceptable process noise standard deviation (Case 2 and 4) gives one-step predicted bearing estimates with RMSE peaks close to the maximum acceptable bearing error $\tilde{\theta}_{\max }$. A process noise standard deviation smaller than $\sigma_{w}^{\min }(r)$ would give one-step predicted bearing estimates with RMSE peaks greater than $\tilde{\theta}_{\max }$. When the tracking range is smaller than $7.2 \mathrm{~km}$ (Case 1), the minimum $\mathrm{MMSE}_{\theta+}$, which is obtained with process noise standard deviation defined by (26a), exceeds the constraint of a third of the radar's beamwidth squared. This is reflected in the number of lost tracks in the simulations. Whereas the combinations of tracking range and process noise standard deviation in Case 2 , 3 , and 4 each leads to a lost track (four missed measurements in a row) in less than 0.2 percent of the simulations, the track is lost in more than 30 percent of the simulations with Case 1.

\section{Tracking with optimal beamwidth}

This set of experiments investigates how the choice of beamwidth affects the tracking performance. The considered scenario involves a stationary radar with variable beamwidth that is tracking the target with trajectory according to Figure 6. The target travels toward the radar, which is located at the origin, from an initial range of $100 \mathrm{~km}$ to a final range of $25 \mathrm{~km}$.

The maximum acceptable RMSE in bearing predictions is related to the current beamwidth as $\tilde{\theta}_{\max }=\theta_{\mathrm{BW}} / 3$. The SNR level is assumed to be $17 \mathrm{~dB}$, and the standard deviation of the bearing measurement noise is related to the beamwidth according to (4). Using these parameters together with the 


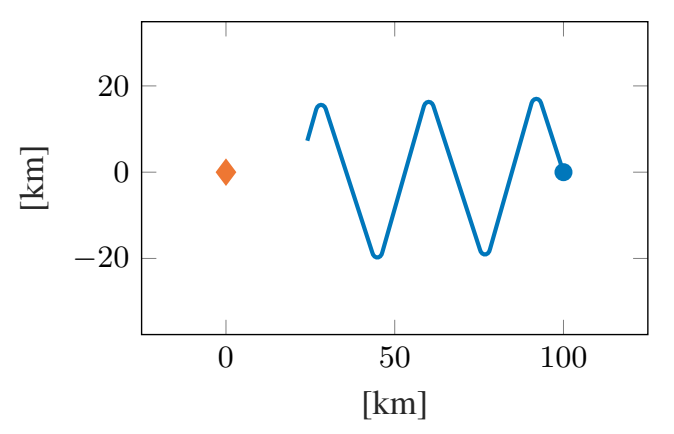

Figure 6: Trajectory of a 6g-maneuvering target that travels at $300 \mathrm{~m} / \mathrm{s}$ toward a radar located at the origin.

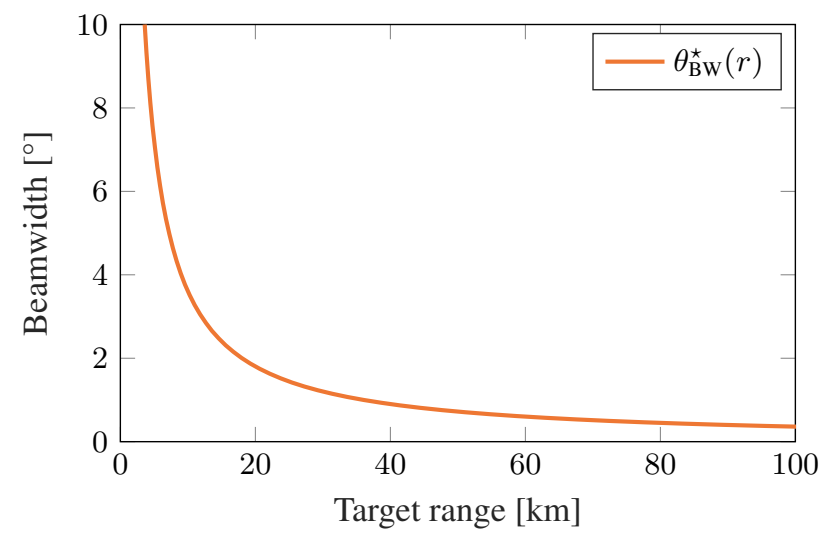

Figure 7: Optimal beamwidth as a function of target range for the scenario depicted in Figure 6.

maximum target acceleration of $A_{\max }=60 \mathrm{~m} / \mathrm{s}^{2}$ and measurement period $T=2 \mathrm{~s}$, the optimal beamwidth can be computed for each tracking range by solving (29) numerically. The result is plotted in Figure 7. Note that Figure 7 also gives the optimal tracking range for each possible beamwidth. The process noise standard deviations that minimize $\mathrm{MMSE}_{p+}^{\mathrm{cr}}$ and satisfy the bearing accuracy requirement are given as functions of the beamwidth for each tracking range in (26).

Table 2 summarizes the tracking parameters used in the simulations. Four different cases are considered: a smaller beamwidth than the optimal with $\sigma_{w}^{\max }$ (Case 5), the optimal beamwidth according to the proposed design method with corresponding optimal process noise standard deviation (Case 6), and a larger beamwidth with both $\sigma_{w}^{\max }$ (Case 7) and $\sigma_{w}^{\min }$ (Case 8). Results from 10000 Monte Carlo simulations are presented in Figure 8.

Figure $8 \mathrm{a}$ shows the errors in the one-step predicted position estimates. As the target gets closer to the sensor, the beamwidth is increased to avoid the risk of the target ending up outside field of view. This increases the noise in the bearing measurements, but the decrease in target range compensates for the increased noise, and the RMSE of the one-step predicted position estimates remains at the same level.

Since decreasing the beamwidth decreases the bearing measurement noise, the smaller beamwidth of Case 5 results in lower RMSE in the position estimates than what is achieved when a larger beamwidth is used (Case 5-8). However, as
Table 2: Parameters for the stationary radar scenario.

\begin{tabular}{lcc}
\hline Case & Beamwidth & Process noise std. dev \\
\hline$\# 5$ & $\frac{1}{2} \theta_{\mathrm{BW}}^{\star}$ & $\sigma_{w}^{\max }\left(\frac{1}{2} \theta_{\mathrm{BW}}^{\star}\right)$ \\
$\# 6$ & $\theta_{\mathrm{BW}}^{\star}$ & $\sigma_{w}^{\max }\left(\theta_{\mathrm{BW}}^{\star}\right)=\sigma_{w}^{\min }\left(\theta_{\mathrm{BW}}^{\star}\right)$ \\
$\# 7$ & $\frac{3}{2} \theta_{\mathrm{BW}}^{\star}$ & $\sigma_{w}^{\max }\left(\frac{3}{2} \theta_{\mathrm{BW}}^{\star}\right)$ \\
$\# 8$ & $\frac{3}{2} \theta_{\mathrm{BW}}^{\star}$ & $\sigma_{w}^{\min }\left(\frac{3}{2} \theta_{\mathrm{BW}}^{\star}\right)$ \\
\hline
\end{tabular}

revealed in Figure 8b, the setup in Case 5 fails to satisfy the bearing accuracy requirement. The RMSE peaks that occur when the target turns are significantly larger than the maximum acceptable bearing error. This leads to a loss of the track in 40 percent of the simulations.

Figures $8 \mathrm{c}$ and $8 \mathrm{~d}$ show the errors in the one-step predicted bearing estimates for Case 6 and Case 7-8, respectively. The method for selecting the process noise standard deviation works as intended, and the RMSE of the predictions during the maneuvers is close to the boundary $\tilde{\theta}_{\max }$ for both Case 6 and Case 8. In Case 6-8, the track is lost in less than 0.2 percent of the simulations.

\section{Conclusions}

This paper has proposed methods to design tracking parameters for range-bearing sensors with limited field of view. Using the results presented in this paper, the tracking range (or beamwidth) and process noise standard deviation can be selected for a given beamwidth (or tracking range) such that the RMSE in the one-step predicted position estimates is minimized during target maneuvers, while ensuring that the target remains within the sensor's field of view with a userdefined probability. The findings have been verified using Monte Carlo simulations.

\section{ACKNOWLEDGMENTS}

This work was partially supported by the Wallenberg AI, Autonomous Systems and Software Program (WASP) funded by the Knut and Alice Wallenberg Foundation.

\section{REFERENCES}

[1] S. Blackman and R. Popoli, Design and Analysis of Modern Tracking Systems. Norwood, MA, USA: Artech house, 1999.

[2] Y. Bar-Shalom, P. K. Willett, and X. Tian, Tracking and data fusion: A handbook of algorithms. Storrs, CT, USA: YBS publishing, 2011.

[3] S. Patil, G. Kahn, M. Laskey, J. Schulman, K. Goldberg, and P. Abbeel, "Scaling up Gaussian belief space planning through covariance-free trajectory optimization and automatic differentiation," in Algorithmic Foundations of Robotics XI. Cham, Switzerland: Springer, 2015, pp. 515-533.

[4] W. D. Blair, "Design of nearly constant velocity track filters for tracking maneuvering targets," in Proceedings of the 11th International Conference on Information Fusion, Cologne, Germany, 2008, pp. 1-7. 


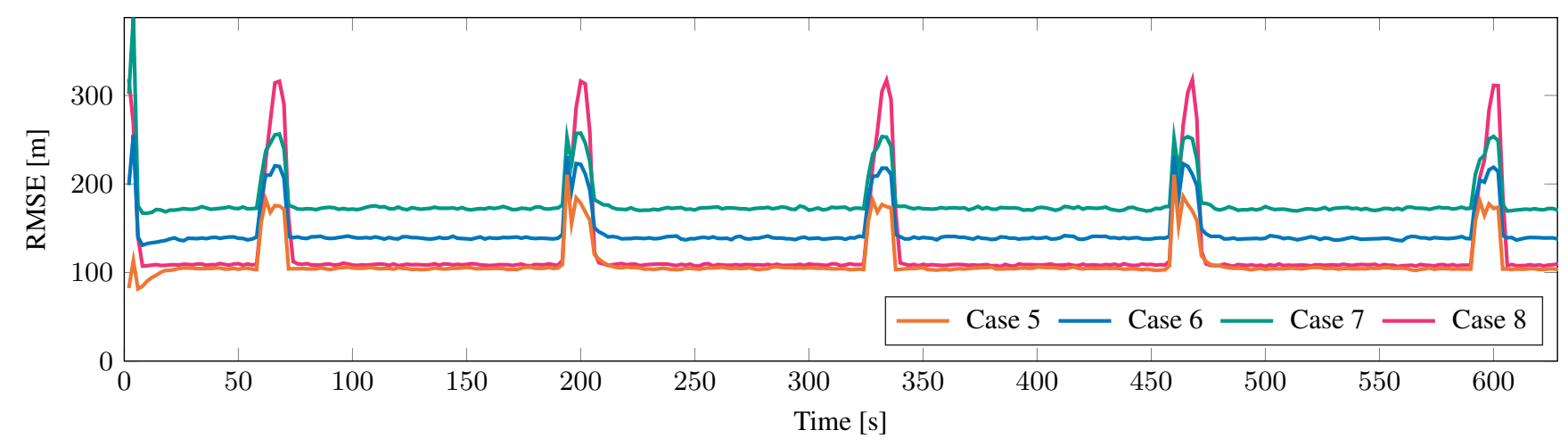

(a) RMSE of the one-step predicted position estimates.

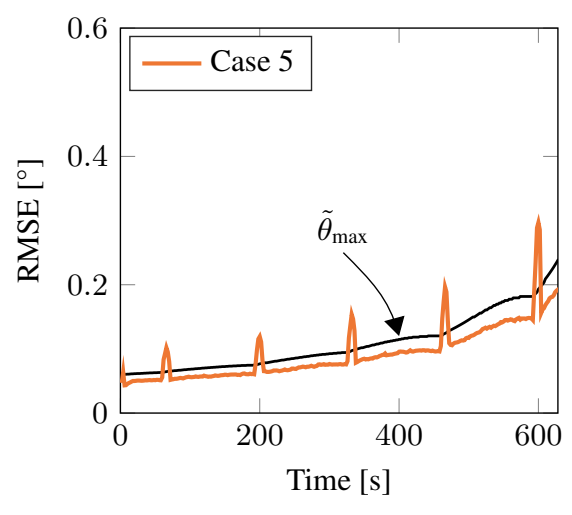

(b)

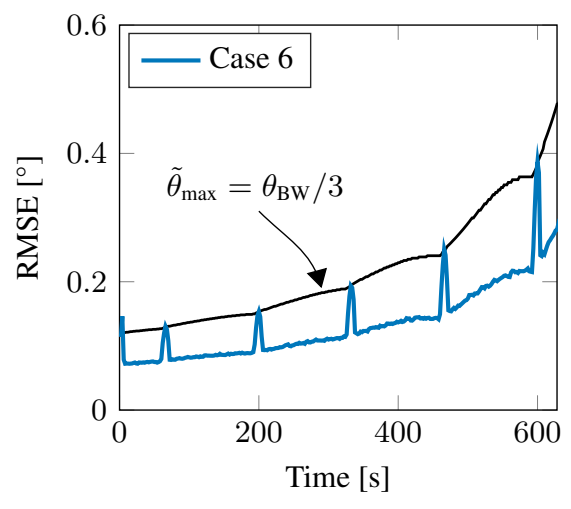

(c)

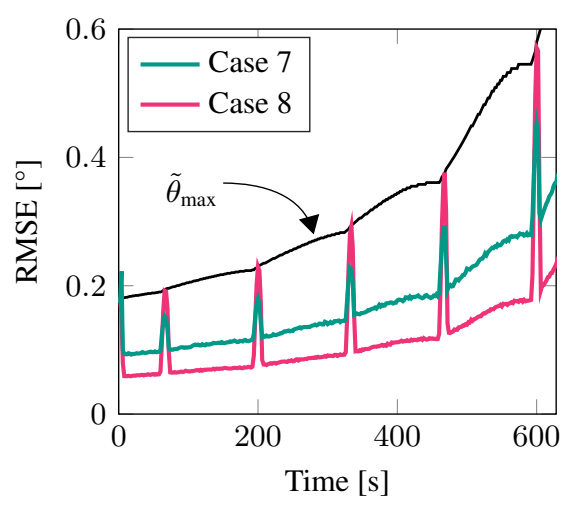

(d)

Figure 8: Results from 10000 Monte Carlo simulations of the scenario in Figure 6 with variable beamwidth and different process noise standard deviations. Figures (b)-(d) show the RMSE of the one-step predicted bearing estimates. The black lines in (b)-(d) correspond to the maximum acceptable bearing error, which is defined as a third of the current beamwidth.

[5] — , "Design of nearly constant velocity filters for radar tracking of maneuvering targets," in Proceedings of IEEE Radar Conference, Atlanta, GA, USA, 2012, pp. 1008-1013.

[6] Y. Wang and W. D. Blair, "Note on sensor resource allocations: Higher rate or better measurements?" in Proceedings of IEEE Aerospace Conference, Big Sky, MT, USA, 2019.

[7] N. Atanasov, J. Le Ny, K. Daniilidis, and G. J. Pappas, "Information acquisition with sensing robots: Algorithms and error bounds," in Proceedings of IEEE International Conference on Robotics and Automation, Hong Kong, China, 2014, pp. 6447-6454.

[8] B. Schlotfeldt, D. Thakur, N. Atanasov, V. Kumar, and G. J. Pappas, "Anytime planning for decentralized multirobot active information gathering," IEEE Robot. Autom. Lett., vol. 3, no. 2, pp. 1025-1032, 2018.

[9] P. Boström-Rost, D. Axehill, and G. Hendeby, "Informative path planning for active tracking of agile targets," in Proceedings of IEEE Aerospace Conference, Big Sky, MT, USA, 2019.

[10] W. D. Blair and M. Brandt-Pearce, "Statistical description of monopulse parameters for tracking rayleigh targets," IEEE Trans. Aerosp. Electron. Syst., vol. 34, no. 2, pp. 597-611, 1998.

[11] J. D. Glass, W. D. Blair, and Y. Bar-Shalom, "Optimiz- ing radar signal to noise ratio for tracking maneuvering targets," in Proceedings of the 17th International Conference on Information Fusion, Salamanca, Spain, 2014.

[12] A. H. Jazwinski, Stochastic Processes and Filtering Theory. New York, NY, USA: Academic Press, 1970.

[13] R. E. Kalman, "A new approach to linear filtering and prediction problems," Transactions of the ASME, Journal of Basic Engineering, vol. 82, no. 1, pp. 35-45, 1960.

[14] P. R. Kalata, "The tracking index: A generalized parameter for $\alpha-\beta$ and $\alpha-\beta-\gamma$ target trackers," IEEE Transactions on Aerospace and Electronic Systems, no. 2, pp. 174-182, 1984.

[15] W. D. Blair and P. Miceli, "Performance prediction of multisensor tracking systems for single maneuvering targets." Journal of Advances in Information Fusion, vol. 7, no. 1, pp. 28-45, 2012. 


\section{BIOGRAPHY}

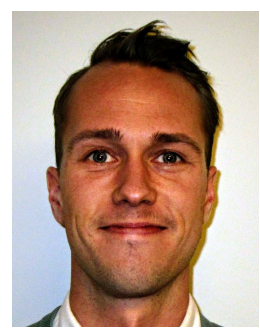

Per Boström-Rost is a Ph.D. student in the Division of Automatic Control, Department of Electrical Engineering, Linköping University. He received his M.Sc. degree in Applied Physics and Electrical Engineering in 2013 and his Lic.Eng. in Electrical Engineering in 2019, both from Linköping University. His research interests include sensor fusion and sensor control.

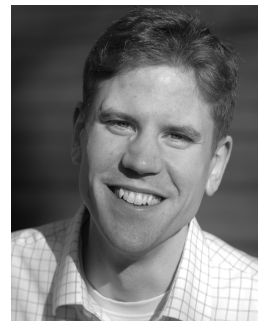

Daniel Axehill received his M.Sc. degree in Applied Physics and Electrical Engineering in 2003. Furthermore, he received the degree of Lic.Eng. in Automatic Control in 2005 and the Ph.D. degree in Automatic Control in 2008. All three degrees are from Linköping University, Linköping, in Sweden. In year 2006 he spent three months at UCLA in Los Angeles. From January 2009 and until November 2010 he worked as a post-doc at the Automatic Control Laboratory at ETH Zurich. He is currently employed as an Associate Professor at the Division of Automatic Control at Linköping University. His research interests are related to optimization, optimal control, motion planning, hybrid systems, and applications of control.

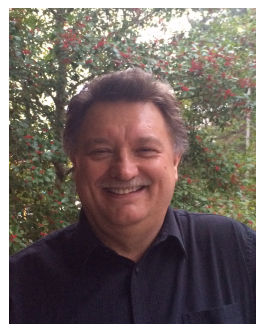

W. D. Blair is a principal research engineer with the Georgia Tech Research Institute (GTRI) and a GTRI Fellow. He is senior member of the Sensors and Electromagnetic Applications Laboratory (SEAL) staff and supports the lab as a subject matter expert in multisensor multitarget tracking, radar data processing, data fusion, modeling and simulation, and algorithm assessment. He received the $B S$ and $M S$ degrees in electrical engineering from Tennessee Technological University in 1985 and 1987. In January 1998, he received the Ph.D. degree in electrical engineering from the University of Virginia. Prior to joining GTRI, Dr. Blair was with the Naval Surface Warfare Center, Dahlgren Division (NSWCDD) in Dahlgren, Virginia. Dr. Blair is a Fellow of the IEEE and recipient of the 2001 IEEE Nathanson Award for Outstanding Young Radar Engineer. He served as the Editor for Radar Systems for IEEE Transactions on Aerospace and Electronic and Systems (T-AES) 199699 and Editor-In-Chief (EIC) for IEEE T-AES from 19992005. Dr. Blair also served on the Board of Governors of IEEE Aerospace and Electronic Systems Society (AESS) from 1998-2003, 2005-2010, and 2012-2017. Dr. Blairs research is reported in over two hundred articles which include 38 refereed journal articles. Dr. Blair is coeditor and coauthor of the book, Multitarget-Multisensor Tracking: Advances and Applications III, and the coauthor of chapter 18 Radar Measurements and author of chapter 19 Radar Tracking Algorithms in Principles of Modern Radar: Basic Principles.

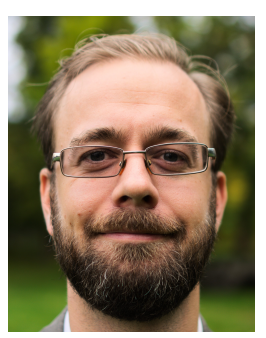

Gustaf Hendeby is Associate Professor in the Division of Automatic Control, Department of Electrical Engineering, Linköping University. He received his MSc in Applied Physics and Electrical Engineering in 2002 and his PhD in Automatic Control in 2008, both from Linköping University. He worked as Senior Researcher at the German Research Center for Artificial Intelligence (DFKI) 2009-2011, and Senior Scientist at Swedish Defense Research Agency (FOI) and held an adjunct Associate Professor position at Linköping University 2011-2015. Dr. Hendeby's main research interests are stochastic signal processing and sensor fusion with applications to nonlinear problems, target tracking, and simultaneous localization and mapping (SLAM). He has experience of both theoretical analysis as well as implementation aspects. 